\title{
Large thermal expansion leads to negative thermo-optic coefficient of halide perovskite $\mathrm{CH}_{3} \mathrm{NH}_{3} \mathrm{PbCl}_{3}$
}

\author{
Taketo Handa $\odot$, Hirokazu Tahara $\odot$, Tomoko Aharen, Ai Shimazaki, Atsushi Wakamiya $\odot$, and Yoshihiko Kanemitsu $\odot^{*}$ \\ Institute for Chemical Research, Kyoto University, Uji, Kyoto 611-0011, Japan
}

(Received 3 April 2020; revised 9 June 2020; accepted 24 June 2020; published 20 July 2020)

\begin{abstract}
Lead halide perovskites have emerged as new optoelectronic materials owing to their outstanding optical properties. There has been increased interest in their temperature-sensitive optical properties and new optical applications have been proposed thereby. Here, we report the origin of the unusual negative thermo-optic coefficient of the halide perovskite $\mathrm{CH}_{3} \mathrm{NH}_{3} \mathrm{PbCl}_{3}$, i.e., a decrease in the refractive index by an increase in temperature. From the temperature dependences of the absorption spectrum and the lattice constant and using the Lorentz oscillator model, we conclude that the negative thermo-optic coefficient below the absorption edge is predominantly determined by the large thermal expansion coefficient inherent to this soft material system. This work demonstrates that the negative thermo-optic coefficient is a distinctive phenomenon reflecting the unique electronic and lattice properties of halide perovskites.
\end{abstract}

DOI: 10.1103/PhysRevMaterials.4.074604

\section{INTRODUCTION}

Lead halide perovskites have attracted attention as a promising class of materials for optoelectronics [1,2]. Earlier work has shown that these materials exhibit superior optoelectronic properties similar to those of high-quality crystalline inorganic semiconductors such as GaAs. Examples of their properties include a sharp absorption edge, large absorption coefficients, high-photoluminescence quantum efficiencies, and long carrier diffusion lengths [3-9]. Taking advantage of these features in conjunction with their facile solution processability, highly efficient solar cells and light-emitting diodes have been demonstrated [10-13], and the applications have been extended to nonlinear optics, such as high-order harmonic generation and optical modulation [14-16].

More recently, growing attention has been paid to the intriguing temperature dependences of their optical properties, which are quite different from those of conventional inorganic semiconductors. Their characteristic thermal properties and optical responses sensitive to temperature variation have been revealed and exploited in novel optical applications [17-22]. As one of them, it was revealed that the halide perovskite exhibits a distinct decrease in refractive index with increasing temperature, i.e., a large negative thermo-optic coefficient [22]. This behavior is rather unique; it is opposite to the positive thermo-optic coefficients usually observed in conventional inorganic semiconductors (e.g., GaAs and $\mathrm{Si}$ ). The origin of the opposite behavior should be related to the

\footnotetext{
*kanemitu@ scl.kyoto-u.ac.jp

Published by the American Physical Society under the terms of the Creative Commons Attribution 4.0 International license. Further distribution of this work must maintain attribution to the author(s) and the published article's title, journal citation, and DOI.
}

inherent electronic structure and optical properties of halide perovskites.

Previous studies have reported that halide perovskites show a blueshift in optical absorption-edge energy with temperature [4,23], which is contrary to the redshift observed in conventional inorganic semiconductors [24]. This opposite shift in the absorption edge may have an impact on the sign of the thermo-optic coefficient. However, the relation between the thermally induced changes in the refractive index and the absorption edge has not been elucidated yet for halide perovskites. Furthermore, this material system possesses unusual thermophysical properties [25-28], originating from the soft metal-halogen framework: halide perovskites are a unique class of crystalline solids and their thermal and optical properties are currently under intense investigation [29-31]. Detailed analyses regarding the thermo-optic coefficients provide a better understanding of their intrinsic thermal and optical properties.

In this paper, we report that the large thermal expansion inherent to this soft material system leads to the negative thermo-optic coefficient of the halide perovskite $\mathrm{MAPbCl}_{3}$ $\left(\mathrm{MA}=\mathrm{CH}_{3} \mathrm{NH}_{3}\right.$ ), by comparing the temperature dependences of the optical absorption spectrum and the lattice constant and performing an analysis based on the Lorentz oscillator model. The expression based on the Lorentz oscillator model describes that the temperature dependence of the refractive index is determined by a thermal expansion coefficient and a temperature-dependent shift in the absorption peak energy of a material. It is shown that the absorption peak energy of $\mathrm{MAPbCl}_{3}$ slightly blueshifts with increasing temperature, which determines the thermo-optic coefficient near the absorption edge. For photon energies lower than the absorption edge, the thermo-optic coefficient is rather determined by the characteristic large thermal expansion coefficient of halide perovskite materials. Both the blueshift of the absorption peak energy and the large thermal expansion are 
the features that result in a negative thermo-optic coefficient. The present work demonstrates that the negative thermo-optic coefficient of halide perovskites strongly reflects the unique thermophysical properties of this class of materials.

\section{EXPERIMENTAL}

\section{A. Samples}

A spin-coating method [32] was used to fabricate $\mathrm{MAPbCl}_{3}$ thin film on quartz substrate. A mixed solution of $N, N$-dimethylformamide and dimethyl sulfoxide, containing equimolar $\mathrm{MACl}$ and $\mathrm{PbCl}_{2}$ was prepared. The precursor solution was dropped on a cleaned substrate. This was followed by spin coating and a toluene (antisolvent) drip before the spinning stopped. The substrate was then annealed on a hot plate, and $\mathrm{MAPbCl}_{3}$ thin film was obtained. The thickness of the film was determined to be $100 \mathrm{~nm}$ from a scanning electron microscope image. The $\mathrm{MAPbCl}_{3}$ single crystals were prepared using an antisolvent vapor-assisted crystallization method as described elsewhere [22,33].

\section{B. Optical measurements}

We measured the transmission and reflectance spectra of the $\mathrm{MAPbCl}_{3}$ thin film, from which the absorption spectrum was obtained. For these measurements, monochromatic light generated from a broadband white light source (EQ-99, Energetiq Technology) and a monochromator (M25, Bunkoukeiki) were used. The full width at half maximum of the light was about $0.2 \mathrm{~nm}$. The light was nearly normal incidence. The light after the output slit of the monochromator was modulated by an optical chopper, and the synchronized signals were recorded using a lock-in amplifier (SR830, Stanford Research Systems). The sample temperature was controlled with a heater and a liquid He flow. The refractive index was determined by measuring the incident-angle dependence of the reflectivity, as described in Ref. [22]. In short, the reflectivity at the reflection angle $\theta$ of the $p$-polarized light with an incidence angle $\theta$ was measured. The refractive index was determined by fitting the obtained incident-angle dependence of the reflectivity with the Fresnel equation for $p$-polarized light [34].

\section{Single-crystal $x$-ray measurement}

The temperature dependence of the lattice constant was determined by the single-crystal x-ray diffraction measurement; the data were collected on a Bruker single-crystal chargecoupled-device $x$-ray diffractometer (SMART APEX II) with Mo $\mathrm{K} \alpha$ radiation $(\lambda=0.71073 \AA)$ and graphite monochromator using a nitrogen gas cooling system (DX-CS190LD, Japan Thermal Engineering).

\section{THEORETICAL BACKGROUND AND EXPERIMENTAL RESULTS}

\section{A. Theoretical background: Lorentz oscillator model}

First, we describe the Lorentz oscillator model, which is a phenomenological model widely used to discuss the thermo-optic coefficient, i.e., the temperature derivative of the refractive index. This model is a simple but effective means to grasp the physical origin of the optical dispersion and its temperature derivative, and it has been employed for various conventional semiconductors $[24,35,36]$. In the present work, we used it to discuss the origin of the negative thermo-optic coefficient of $\mathrm{MAPbCl}_{3}$. Here, the refractive index $n$ below the fundamental absorption edge is given by [37]

$$
n=\sqrt{1+\sum_{i} \frac{A_{i} E_{\mathrm{p}}^{2}\left(E_{i}^{2}-E^{2}\right)}{\left(E_{i}^{2}-E^{2}\right)^{2}+\left(\Gamma_{i} E\right)^{2}}}
$$

where $E$ represents the photon energy, and $E_{i}, \Gamma_{i}$, and $A_{i}$ represent the resonance energy, the broadening parameter, and the oscillator strength of the $i$ th transition, respectively. The plasma energy $E_{\mathrm{p}}$ is a function of the number of valence electrons per unit volume $(N / V): E_{\mathrm{p}}=\left(N e^{2} \hbar^{2} / \epsilon_{0} m V\right)^{1 / 2}$, where $\epsilon_{0}$ is the vacuum permittivity, $e$ is the elementary charge, $m$ is the mass of the oscillator, and $\hbar$ is the reduced Planck constant.

The temperature derivative of the refractive index, i.e., the thermo-optic coefficient, is obtained by differentiating Eq. (1) with respect to temperature:

$$
\frac{d n}{d T}=\frac{n_{0}^{2}-1}{2 n}\left(\sum_{i} \frac{A_{i}}{E_{i}^{2}}\right)^{-1} \sum_{i} A_{i}\left[-3 \alpha \frac{E_{i}^{2}-E^{2}}{\left(E_{i}^{2}-E^{2}\right)^{2}+\left(\Gamma_{i} E\right)^{2}}-2 \frac{1}{E_{i}} \frac{d E_{i}}{d T} \frac{E_{i}^{2}\left\{\left(E_{i}^{2}-E^{2}\right)^{2}-\left(\Gamma_{i} E\right)^{2}\right\}}{\left\{\left(E_{i}^{2}-E^{2}\right)^{2}+\left(\Gamma_{i} E\right)^{2}\right\}^{2}}\right] .
$$

Note that Eq. (2) contains a term related to the linear thermal expansion coefficient $\alpha$. This is because the plasma energy $E_{\mathrm{p}}$ is a function of the valence electron density, and its temperature derivative is given by $d E_{\mathrm{p}}^{2} / d T=-E_{\mathrm{p}}^{2}(1 / V)(d V / d T)=$ $-3 \alpha E_{\mathrm{p}}^{2}$. Here, we applied the relation between the volumetric expansion coefficient $\alpha_{\mathrm{V}}$ and linear expansion coefficient $\alpha$ $\left(\alpha_{\mathrm{V}}=3 \alpha\right)$, since $\mathrm{MAPbCl}_{3}$ possesses a cubic structure near room temperature [38]. Additionally, in the limit $E \rightarrow 0$ in Eq. (1), $E_{\mathrm{p}}$ can be written using the long-wavelength refractive index, $n_{0}=n(E \rightarrow 0): E_{\mathrm{p}}^{2}=\left(n_{0}^{2}-1\right)\left(\sum_{i} A_{i} / E_{i}^{2}\right)^{-1}$. Using these relations, we obtained Eq. (2). Equation (2) means that the thermo-optic coefficient of a material is determined by the thermally induced change in the valence electron density and the thermal shift of the resonance energy.

The general properties of the thermo-optic coefficient can be deduced from Eq. (2); (i) the temperature-induced lattice expansion $(\alpha>0)$ results in a decrease in the refractive index $(d n / d T<0)$, and (ii) the blueshift of the resonance energy $\left(d E_{i} / d T>0\right)$ also results in the decrease in the refractive index $(d n / d T<0)$. In conventional inorganic semiconduc- 

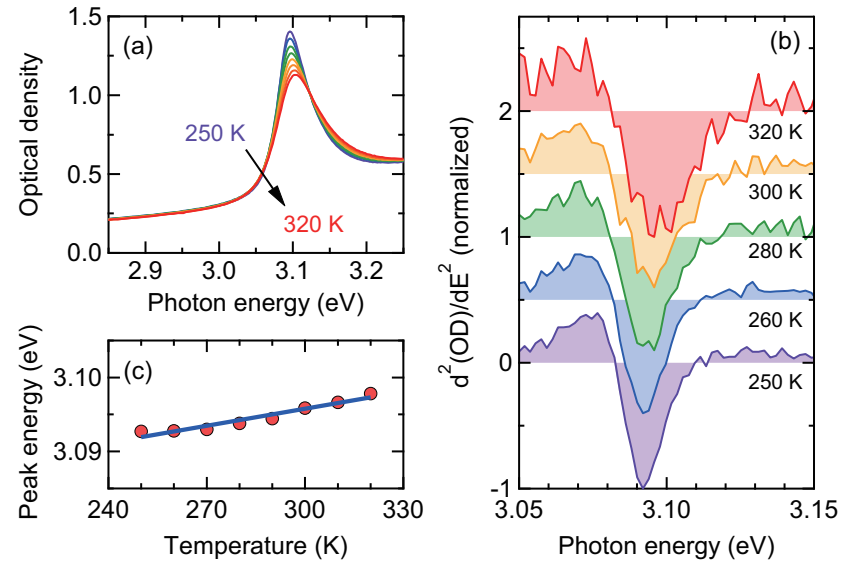

FIG. 1. Temperature dependence of the exciton energy of $\mathrm{MAPbCl}_{3}$. (a) Absorption spectra of the $\mathrm{MAPbCl}_{3}$ thin film at temperatures ranging from 250 to $320 \mathrm{~K}$. (b) Second derivatives of the absorption spectra. The spectra are normalized and offset for clarity. (c) Temperature dependence of the peak energy. The solid line shows a fitting result using a linear function.

tors such as $\mathrm{GaAs}$ and $\mathrm{Si}$, the value of the linear expansion coefficient $\alpha$ is small and the thermal shift of the bandgap energy determines the thermo-optic coefficient [39]. In these semiconductors, the band-gap energy decreases with temperature $\left(d E_{i} / d T<0\right)$ [24], thus, they possess positive thermo-optic coefficients $(d n / d T>0)$. On the other hand, halide perovskites featuring a soft lattice nature [27,29,40,41] show a large thermal expansion; e.g., the reported values of linear expansion coefficients of some perovskite samples are one order larger than those in conventional semiconductors such as GaAs and Si [42-44]. Therefore, in halide perovskites, the thermal expansion effect should have a large impact on the thermo-optic coefficient. In the present work, for quantitative analysis, the thermal expansion coefficient of $\mathrm{MAPbCl}_{3}$ is determined precisely via single-crystal x-ray diffraction as in the following section. In addition, a thermal shift of the resonance energy is needed for the analysis [see Eq. (2)]. However, in the wide-gap semiconductor $\mathrm{MAPbCl}_{3}$, the temperature dependence of the fundamental absorption peak energy (exciton energy) has not been elucidated, and its impact on the thermo-optic coefficient is elusive. Through the experimental determination of the thermal shift of the exciton energy and the thermal expansion coefficient and the analysis based on the Lorentz oscillator model, we will discuss the origin of the thermo-optic coefficient in $\mathrm{MAPbCl}_{3}$.

\section{B. Temperature dependences of the absorption spectrum, lattice constant, and refractive index}

Figure 1(a) shows the absorption spectra of $\mathrm{MAPbCl}_{3}$ in the temperature range from 250 to $320 \mathrm{~K}$. These absorption spectra were obtained by simultaneously measuring the transmittance and reflectance spectra of a $\mathrm{MAPbCl}_{3}$ thin film. A sharp absorption peak is observed at a photon energy of $\sim 3.1 \mathrm{eV}$, which is due to the large exciton binding energy of this material [45]. Note that the temperature range studied here is sufficiently above the cubic to tetragonal phasetransition temperature of $\mathrm{MAPbCl}_{3}, 177.2 \mathrm{~K}$ [46]. Since

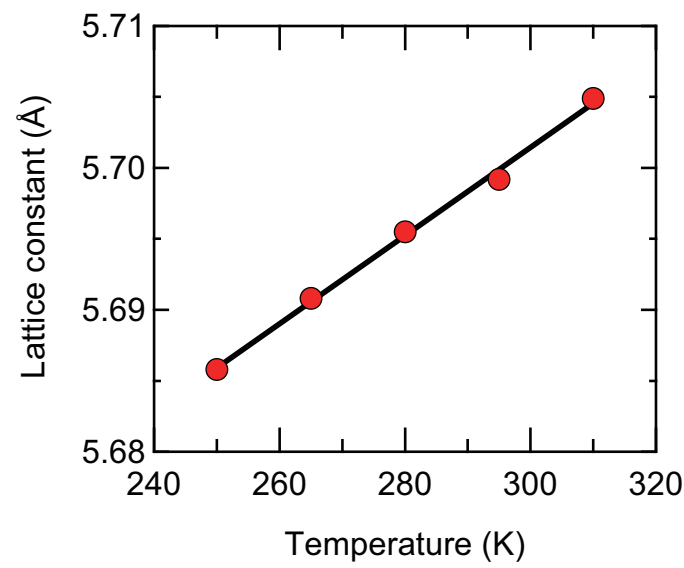

FIG. 2. Temperature dependence of the lattice constant of a cubic $\mathrm{MAPbCl}_{3}$ single crystal. The solid line shows a fitting result using a linear function.

complicated phenomena such as two-phase coexistence and thermal hysteresis appear at around the phase-transition temperature, we use the data in the high-temperature region and discuss the intrinsic optical properties in the cubic phase.

The exciton absorption peak energy was determined as the second derivative minima [47] of the absorption spectrum [Fig. 1(b)]. The temperature dependence of the obtained exciton energy is shown in Fig. 1 (c). $\mathrm{MAPbCl}_{3}$ shows a blueshift in the absorption peak energy with temperature. For this temperature range, the magnitude of the thermal shift was determined to be $d E_{\text {abs }} / d T=7.7 \times 10^{-5}\left(\mathrm{eV} \mathrm{K}^{-1}\right)$ [blue solid line in Fig. 1(c)]. The blueshift of the absorption peak energy is opposite to those in conventional inorganic semiconductors [24] and is the characteristic resulting in the negative thermo-optic coefficient [see Eq. (2)]. The same trend has also been reported in iodide and bromide perovskites $[4,23]$. The blueshift observed in halide perovskites can be attributed to thermal expansion and electron-phonon interactions [17,48]. In this way, we have determined the thermal shift of the exciton peak energy and thus the contribution of the second term on the right-hand side of Eq. (2).

Figure 2 shows the temperature dependence of the lattice constant of $\mathrm{MAPbCl}_{3}$ determined by the single-crystal x-raydiffraction technique. The measurement temperature range was around room temperature, where $\mathrm{MAPbCl}_{3}$ is known to have a cubic structure as discussed above [46]. The obtained data were well explained by the cubic $P m \overline{3} m$ structure $[38,49]$ and the lattice constant at each temperature was determined as shown in Fig. 2. From the temperature dependence, the linear thermal expansion coefficient of $\mathrm{MAPbCl}_{3}$ was evaluated to be $\alpha=5.45 \times 10^{-5}\left(\mathrm{~K}^{-1}\right)$, confirming its large thermal expansion behavior. This value is in agreement with that determined by an optical measurement [22].

Figure 3 shows the temperature dependence of the refractive indices of $\mathrm{MAPbCl}_{3}$ at different photon energies. These can be determined using the method described in Ref. [22]. The photon energies presented here are below the fundamental absorption edge of $\mathrm{MAPbCl}_{3}$ (see Fig. 1). In this transparent wavelength region, one can confirm normal dispersion (a larger refractive index for higher photon energy). Figure 3 


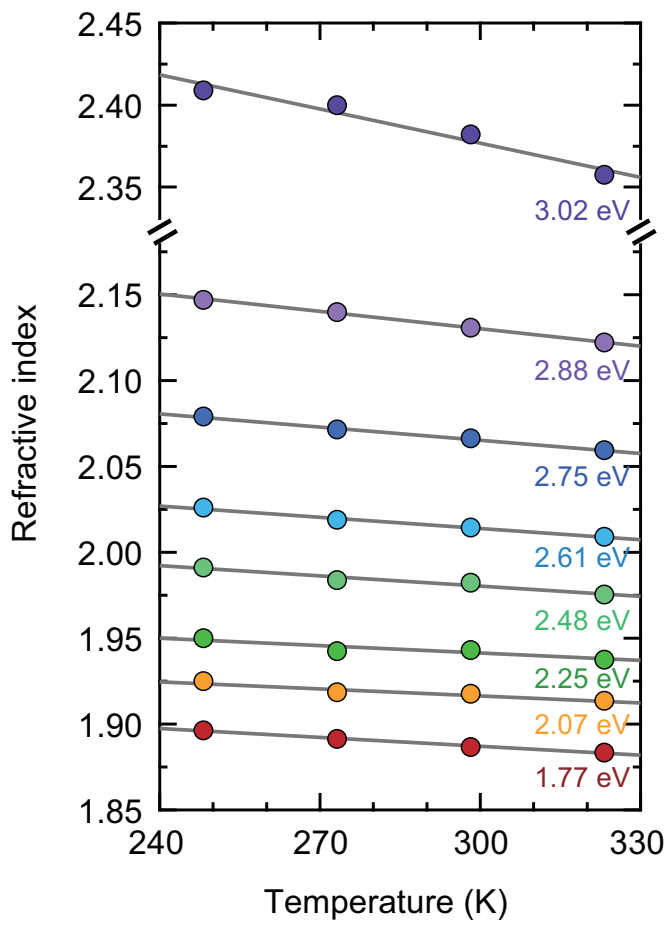

FIG. 3. Temperature dependence of the refractive indices of $\mathrm{MAPbCl}_{3}$ at different photon energies. These photon energies are smaller than the fundamental absorption energy of $\mathrm{MAPbCl}_{3}$. The gray solid lines show a fitting result using a linear function, from which the thermo-optic coefficient is obtained.

clearly demonstrates that the refractive index at each photon energy decreases with increasing temperature, i.e., a negative thermo-optic coefficient [22]. The temperature dependence was fitted by a linear function, and the obtained slope gives the thermo-optic coefficient $d n / d T$ (the fitting results are shown as gray solid lines in Fig. 3). The halide perovskite $\mathrm{MAPbCl}_{3}$ has negative thermo-optic coefficients in the visible wavelength region, and its magnitude increases near the absorption edge.

Next, we determined the long-wavelength refractive index $n_{0}$ that appears in Eq. (2). The value of $n_{0}$ can be determined experimentally via the method described in Ref. [50]. Specifically, the function $\left(n^{2}-1\right)^{-1}$ is plotted against the square of the photon energy $E^{2}$ and extrapolation $\left(E^{2} \rightarrow 0\right)$ gives $\left(n_{0}^{2}-1\right)^{-1}$. The value of $n_{0}$ can be determined by analyzing the values well below the absorption edge, since the contribution from the excitonic transition exists near the absorption edge [50]. This analysis is performed in Fig. 4 for the refractive index at $298 \mathrm{~K}$, from which we obtained the value of the long-wavelength refractive index: $n_{0}=1.80$.

\section{DISCUSSION}

By placing the experimentally determined values in Eq. (2), we can discuss the origin of the thermo-optic coefficient of $\mathrm{MAPbCl}_{3}$. In Eq. (2), it can be complicated to consider multiple oscillators. Although there has been a report on using an empirical model that considers only one average energy of two resonances for simplicity [51], such a simplified description makes it difficult to quantitatively discuss the

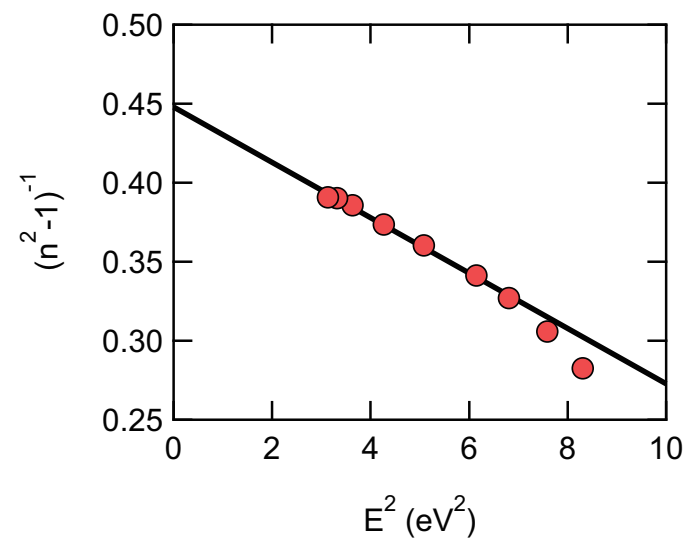

FIG. 4. Plot of $\left(n^{2}-1\right)^{-1}$ versus the square of the photon energy, where $n$ represents the refractive index of $\mathrm{MAPbCl}_{3}$ at $298 \mathrm{~K}$. By extrapolating the data to $E^{2}=0$, the long-wavelength refractive index was determined to be $n_{0}=1.80$.

contribution of the peak energy shift and the thermal expansion effect in halide perovskites. Instead, we will employ a relatively strict model considering two predominant resonance energies $(i=1,2)$ to comprehensively discuss the dispersion of the thermo-optic coefficient. The first resonance is the exciton transition at the fundamental absorption edge with a photon energy $E_{1}=3.1 \mathrm{eV}(i=1)$. As shown in Fig. 3, the magnitude of the thermo-optic coefficient becomes larger near the absorption edge, indicating a crucial contribution from the excitonic transition. The second resonance is the strong absorption transition with a broad linewidth located at a higher photon energy $E_{2}=5.28 \mathrm{eV}(i=2)$ [52,53]. Whereas other peaks and the continuous state above the fundamental absorption edge exist, the transition at $5.28 \mathrm{eV}$ has a relatively strong oscillator strength and should play a critical role among them. The transitions at $E_{1}$ and $E_{2}$ both originate from the direct transition between the same valence and conduction bands ( $E_{1}$ occurs at the $R$ point, and $E_{2}$ occurs at the $X$ point) [52]; therefore, we can consider that these transitions show a similar value of the thermal shift in peak energy. Based on these discussions, the analysis of the thermo-optic coefficient of $\mathrm{MAPbCl}_{3}$ was performed.

The experimental values of the thermo-optic coefficient are shown in Fig. 5 as red filled circles, which were determined from the fitting in Fig. 3. In order to analyze these data by using Eq. (2), we used $E_{1}=3.096 \mathrm{eV}$ (Fig. 1) and $\Gamma_{1}=$ $0.07 \mathrm{eV}$ determined in the reflectance measurement and $\alpha=$ $5.45 \times 10^{-5}\left(\mathrm{~K}^{-1}\right)$ (Fig. 2) from the x-ray-diffraction measurement. The thermal shift of the $E_{1}$ peak was determined from Fig. 1(c): $d E_{1} / d T=7.7 \times 10^{-5}\left(\mathrm{eV} \mathrm{K}^{-1}\right)$. The peak energy and the broadening parameter of the second oscillator were taken from the literature: $E_{2}=5.28 \mathrm{eV}$ and $\Gamma_{2}=1.42$ $\mathrm{eV}[52,53]$. The sum of the amplitudes of these oscillators is held to be $A_{1}+A_{2}=1$. We considered $d E_{1} / d T=d E_{2} / d T$ for the reason mentioned above [52]. Note that the experimental results cannot be explained when we only consider the $E_{1}$ transition. Since the $E_{2}$ transition has the large amplitude as shown below, this transition and its change due to the thermal expansion should be considered for accurate analysis of the optical dispersion. 


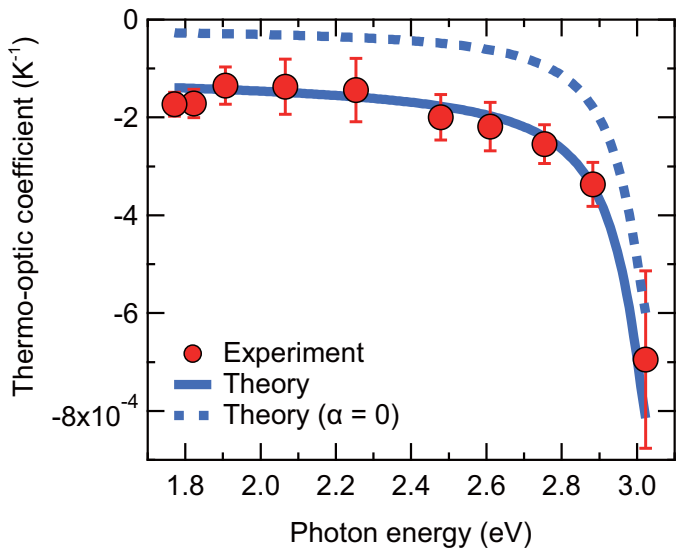

FIG. 5. Analysis of the thermo-optic coefficient $d n / d T$ of $\mathrm{MAPbCl}_{3}$. The red filled circles show the experimental values determined from Fig. 3. The blue solid curve shows the fitting result using Eq. (2), while the blue dotted curve represents the calculation without the contribution of the thermal expansion.

We performed a fitting of the experimental data using Eq. (2), where only $A_{1}$ was an adjustable parameter. The best-fit result is shown with the blue solid line in Fig. 5 for $A_{1}=0.05$. This result shows that the Lorentz oscillator model well explains the dispersion of the experimental thermo-optic coefficient. Here, a relatively small contribution of the $E_{1}$ peak $\left(A_{1}=0.05 \ll A_{2}\right)$ was obtained. This is attributed to the broadening parameter of the $E_{1}$ peak $\left(\Gamma_{1}=0.07 \mathrm{eV}\right)$ being much smaller than that of the $E_{2}$ peak $\left(\Gamma_{2}=1.42 \mathrm{eV}\right)$ and the effective oscillator strength of the latter being larger. While the effective amplitude of the $E_{1}$ transition is small, it has an impact for the energy region near $E_{1}$ and therefore leads to the large contribution to the thermo-optic coefficient near the absorption edge.

Furthermore, we plotted the theoretical curve for $\alpha=0$ (the blue dotted line in Fig. 5). This clearly shows that the thermal expansion effect plays a decisive role in determining the thermo-optic coefficient. In particular, the thermo-optic coefficient in the photon energies well below the absorption edge is mainly determined by thermal expansion. The large thermal expansion of halide perovskites can be attributed to the soft nature of the lead-halogen framework [40], which is likely due to the existence of lead and halide ions with large ionic radii and a correspondingly long lead-halogen bond length. These results demonstrate that their characteristic microscopic lattice properties have a strong impact on the macroscopic optical response.

Now let us compare the thermo-optic coefficients of $\mathrm{MAPbCl}_{3}$ and other semiconductors (Fig. 6), to facilitate an intuitive understanding of the uniqueness of halide perovskites. The vertical axis in Fig. 6 represents the normalized thermo-optic coefficient $\left(n^{-1} d n / d T\right)$, while the horizontal axis represents the normalized thermal shift in absorption energy $\left(E_{\text {abs }}^{-1} d E_{\text {abs }} / d T\right)$. The value for $\mathrm{MAPbCl}_{3}$ (red star) was determined in this work, while the other values for various semiconductors were taken from Refs. [39,54,55]. These thermo-optic coefficients are determined for photon energies sufficiently below the fundamental absorption edge (transparent wavelength region).

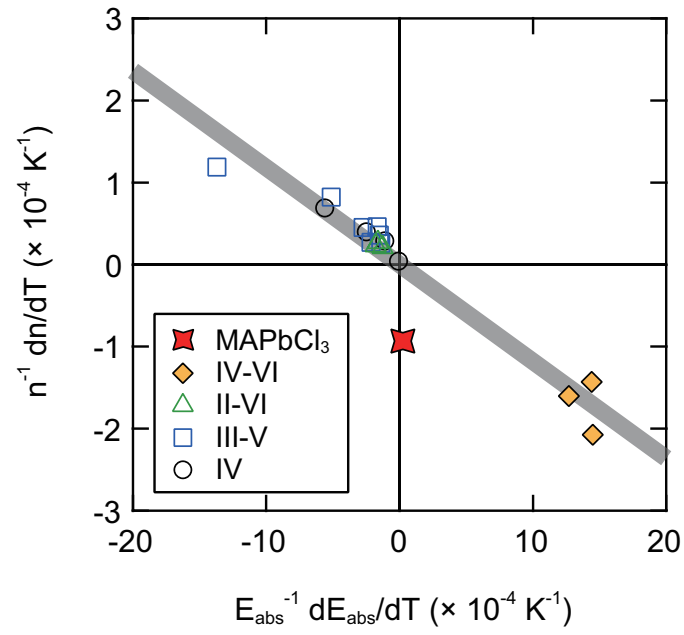

FIG. 6. Comparison of the normalized thermo-optic coefficient and thermal shift of the absorption edge for various semiconductors. The gray line represents a linear function. The value for $\mathrm{MAPbCl}_{3}$ (red star) is determined in this work, while those for other semiconductors are taken from Refs. [39,54,55].

For group IV, III-V, and II-VI semiconductors such as $\mathrm{Si}, \mathrm{GaAs}$, and CdS (the open symbols in Fig. 6), the values gather in the upper left; i.e., they show a thermally induced redshift in the absorption peak and positive thermo-optic coefficients. Although the magnitudes of their thermo-optic coefficients differ, we found that the values lie on one line shown in gray. This means that the thermo-optic coefficients of these semiconductors are determined mainly by the thermal shift of the absorption peak energy. As discussed in the previous section, these conventional semiconductors possess relatively small thermal expansion coefficients $[43,44]$. Thus, the thermal expansion plays a negligible role, while the peak energy shift mainly determines the thermo-optic coefficients [see Eq. (2)]. Group IV-VI semiconductors such as PbS show a trend opposite to that of the above typical semiconductors (the values gather in the lower right area); nevertheless, their values are also on the line in the figure, suggesting that their thermo-optic coefficients are also determined by a thermal shift in absorption energy.

On the other hand, we found that the thermo-optic coefficient of $\mathrm{MAPbCl}_{3}$ (shown with a red star in Fig. 6) deviates remarkably from the linear line. $\mathrm{MAPbCl}_{3}$ shows the large negative thermo-optic coefficient despite the small blueshift of the absorption peak energy. This is a rather unique feature of halide perovskites. This comparison clearly indicates that the different physical mechanisms govern the thermo-optic responses for halide perovskites and conventional semiconductors: the thermal expansion for the former and the resonance energy shift for the latter. Our results demonstrate that halide perovskites are unusual semiconductors in which a large thermal expansion coefficient results in a large negative thermo-optic coefficient.

\section{CONCLUSION}

In summary, we reported the origin of the negative thermooptic coefficient of the halide perovskite $\mathrm{MAPbCl}_{3}$. It is 
shown that the large thermal expansion and blueshift in the absorption peak energy result in the negative thermo-optic coefficient of this material. The blueshift in the exciton peak energy contributes to the large magnitude of the negative thermo-optic coefficient near the absorption edge. The negative thermo-optic coefficient well below the absorption edge rather originates from its large thermal expansion coefficient. This work has revealed that the negative thermo-optic coefficient is a phenomenon strongly reflecting the inherent temperature dependence of the electronic states and the lattice properties of halide perovskites. Overall, the present results shed light on the intrinsic optical properties of halide perovskites and provide essential knowledge for the design of perovskite-based photonic devices and novel applications based on their unique thermo-optic properties.

\section{ACKNOWLEDGMENTS}

Part of this work was supported by JST-CREST (Grant No. JPMJCR16N3) and JSPS KAKENHI (Grant No. 17J09650). The authors thank Y. Hashikawa of Kyoto University for support on the single-crystal x-ray measurement.
[1] S. D. Stranks and H. J. Snaith, Metal-halide perovskites for photovoltaic and light-emitting devices, Nat. Nanotechnol. 10, $391(2015)$

[2] Y. Kanemitsu, Luminescence spectroscopy of lead-halide perovskites: Materials properties and application as photovoltaic devices, J. Mater. Chem. C 5, 3427 (2017).

[3] S. De Wolf, J. Holovsky, S.-J. Moon, P. Löper, B. Niesen, M. Ledinsky, F.-J. Haug, J.-H. Yum, and C. Ballif, Organometallic halide perovskites: Sharp optical absorption edge and its relation to photovoltaic performance, J. Phys. Chem. Lett. 5, 1035 (2014).

[4] Y. Yamada, T. Nakamura, M. Endo, A. Wakamiya, and Y. Kanemitsu, Near-band-edge optical responses of solutionprocessed organic-inorganic hybrid perovskite $\mathrm{CH}_{3} \mathrm{NH}_{3} \mathrm{PbI}_{3}$ on mesoporous $\mathrm{TiO}_{2}$ electrodes, Appl. Phys. Express 7, 032302 (2014).

[5] T. Yamada, Y. Yamada, Y. Nakaike, A. Wakamiya, and Y. Kanemitsu, Photon Emission and Reabsorption Processes in $\mathrm{CH}_{3} \mathrm{NH}_{3} \mathrm{PbBr}_{3}$ Single Crystals Revealed by Time-Resolved Two-Photon-Excitation Photoluminescence Microscopy, Phys. Rev. Appl. 7, 014001 (2017).

[6] I. L. Braly, D. W. deQuilettes, L. M. Pazos-Outón, S. Burke, M. E. Ziffer, D. S. Ginger, and H. W. Hillhouse, Hybrid perovskite films approaching the radiative limit with over $90 \%$ photoluminescence quantum efficiency, Nat. Photonics 12, 355 (2018).

[7] S. D. Stranks, G. E. Eperon, G. Grancini, C. Menelaou, M. J. P. Alcocer, T. Leijtens, L. M. Herz, A. Petrozza, and H. J. Snaith, Electron-hole diffusion lengths exceeding 1 micrometer in an organometal trihalide perovskite absorber, Science $\mathbf{3 4 2}$, 341 (2013).

[8] G. Xing, N. Mathews, S. Sun, S. S. Lim, Y. M. Lam, M. Grätzel, S. Mhaisalkar, and T. C. Sum, Long-range balanced electronand hole-transport lengths in organic-inorganic $\mathrm{CH}_{3} \mathrm{NH}_{3} \mathrm{PbI}_{3}$, Science 342, 344 (2013).

[9] Y. Kanemitsu and T. Handa, Photophysics of metal halide perovskites: From materials to devices, Jpn. J. Appl. Phys. 57, 090101 (2018).

[10] A. Kojima, K. Teshima, Y. Shirai, and T. Miyasaka, Organometal halide perovskites as visible-light sensitizers for photovoltaic cells, J. Am. Chem. Soc. 131, 6050 (2009).

[11] E. H. Jung, N. J. Jeon, E. Y. Park, C. S. Moon, T. J. Shin, T.-Y. Yang, J. H. Noh, and J. Seo, Efficient, stable and scalable perovskite solar cells using poly(3-hexylthiophene), Nature (London) 567, 511 (2019).

[12] Z.-K. Tan, R. S. Moghaddam, M. L. Lai, P. Docampo, R. Higler, F. Deschler, M. Price, A. Sadhanala, L. M. Pazos, D. Credgington, F. Hanusch, T. Bein, H. J. Snaith, and R. H. Friend, Bright light-emitting diodes based on organometal halide perovskite, Nat. Nanotechnol. 9, 687 (2014).

[13] K. Lin, J. Xing, L. N. Quan, F. P. G. de Arquer, X. Gong, J. Lu, L. Xie, W. Zhao, D. Zhang, C. Yan, W. Li, X. Liu, Y. Lu, J. Kirman, E. H. Sargent, Q. Xiong, and Z. Wei, Perovskite lightemitting diodes with external quantum efficiency exceeding 20 per cent, Nature (London) 562, 245 (2018).

[14] H. Tahara, T. Aharen, A. Wakamiya, and Y. Kanemitsu, Photorefractive effect in organic-inorganic hybrid perovskites and its application to optical phase shifter, Adv. Opt. Mater. 6, 1701366 (2018).

[15] H. Hirori, P. Xia, Y. Shinohara, T. Otobe, Y. Sanari, H. Tahara, N. Ishii, J. Itatani, K. L. Ishikawa, T. Aharen, M. Ozaki, A. Wakamiya, and Y. Kanemitsu, High-order harmonic generation from hybrid organic-inorganic perovskite thin films, APL Mater. 7, 041107 (2019).

[16] K. Ohara, T. Yamada, H. Tahara, T. Aharen, H. Hirori, H. Suzuura, and Y. Kanemitsu, Excitonic enhancement of optical nonlinearities in perovskite $\mathrm{CH}_{3} \mathrm{NH}_{3} \mathrm{PbCl}_{3}$ single crystals, Phys. Rev. Mater. 3, 111601(R) (2019).

[17] B. J. Foley, D. L. Marlowe, K. Sun, W. A. Saidi, L. Scudiero, M. C. Gupta, and J. J. Choi, Temperature dependent energy levels of methylammonium lead iodide perovskite, Appl. Phys. Lett. 106, 243904 (2015).

[18] S.-T. Ha, C. Shen, J. Zhang, and Q. Xiong, Laser cooling of organic-inorganic lead halide perovskites, Nat. Photonics 10, 115 (2016).

[19] T. Yamada, T. Aharen, and Y. Kanemitsu, Up-converted photoluminescence from $\mathrm{CH}_{3} \mathrm{NH}_{3} \mathrm{PbI}_{3}$ perovskite semiconductors: Implications for laser cooling, Phys. Rev. Mater. 3, 024601 (2019).

[20] J. Lin, M. Lai, L. Dou, C. S. Kley, H. Chen, F. Peng, J. Sun, D. Lu, S. A. Hawks, C. Xie, F. Cui, A. P. Alivisatos, D. T. Limmer, and P. Yang, Thermochromic halide perovskite solar cells, Nat. Mater. 17, 261 (2018).

[21] S. Yakunin, B. M. Benin, Y. Shynkarenko, O. Nazarenko, M. I. Bodnarchuk, D. N. Dirin, C. Hofer, S. Cattaneo, and M. V. Kovalenko, High-resolution remote thermometry and 
thermography using luminescent low-dimensional tin-halide perovskites, Nat. Mater. 18, 846 (2019).

[22] T. Handa, H. Tahara, T. Aharen, and Y. Kanemitsu, Large negative thermo-optic coefficients of a lead halide perovskite, Sci. Adv. 5, eaax0786 (2019).

[23] N. Sestu, M. Cadelano, V. Sarritzu, F. Chen, D. Marongiu, R. Piras, M. Mainas, F. Quochi, M. Saba, A. Mura, and G. Bongiovanni, Absorption f-sum rule for the exciton binding energy in methylammonium lead halide perovskites, J. Phys. Chem. Lett. 6, 4566 (2015).

[24] P. Y. Yu and M. Cardona, Temperature coefficient of the refractive index of diamond- and zinc-blende-type semiconductors, Phys. Rev. B 2, 3193 (1970).

[25] A. Pisoni, J. Jaćimović, O. S. Barišić, M. Spina, R. Gaál, L. Forró, and E. Horváth, Ultra-low thermal conductivity in organic-inorganic hybrid perovskite $\mathrm{CH}_{3} \mathrm{NH}_{3} \mathrm{PbI}_{3}$, J. Phys. Chem. Lett. 5, 2488 (2014).

[26] G. A. Elbaz, W.-L. Ong, E. A. Doud, P. Kim, D. W. Paley, X. Roy, and J. A. Malen, Phonon speed, not scattering, differentiates thermal transport in lead halide perovskites, Nano Lett. 17, 5734 (2017).

[27] A. C. Ferreira, A. Létoublon, S. Paofai, S. Raymond, C. Ecolivet, B. Rufflé, S. Cordier, C. Katan, M. I. Saidaminov, A. A. Zhumekenov, O. M. Bakr, J. Even, and P. Bourges, Elastic Softness of Hybrid Lead Halide Perovskites, Phys. Rev. Lett. 121, 085502 (2018)

[28] C. Ge, M. Hu, P. Wu, Q. Tan, Z. Chen, Y. Wang, J. Shi, and J. Feng, Ultralow thermal conductivity and ultrahigh thermal expansion of single-crystal organic-inorganic hybrid perovskite $\mathrm{CH}_{3} \mathrm{NH}_{3} \mathrm{PbX}_{3}(\mathrm{X}=\mathrm{Cl}, \mathrm{Br}, \mathrm{I})$, J. Phys. Chem. C 122, 15973 (2018).

[29] O. Yaffe, Y. Guo, L. Z. Tan, D. A. Egger, T. Hull, C. C. Stoumpos, F. Zheng, T. F. Heinz, L. Kronik, M. G. Kanatzidis, J. S. Owen, A. M. Rappe, M. A. Pimenta, and L. E. Brus, Local Polar Fluctuations in Lead Halide Perovskite Crystals, Phys. Rev. Lett. 118, 136001 (2017).

[30] M. Nagai, T. Tomioka, M. Ashida, M. Hoyano, R. Akashi, Y. Yamada, T. Aharen, and Y. Kanemitsu, Longitudinal Optical Phonons Modified by Organic Molecular Cation Motions in Organic-Inorganic Hybrid Perovskites, Phys. Rev. Lett. 121, 145506 (2018).

[31] K. Miyata, T. L. Atallah, and X.-Y. Zhu, Lead halide perovskites: Crystal-liquid duality, phonon glass electron crystals, and large polaron formation, Sci. Adv. 3, e1701469 (2017).

[32] N. J. Jeon, J. H. Noh, Y. C. Kim, W. S. Yang, S. Ryu, and S. Il Seok, Solvent engineering for high-performance inorganicorganic hybrid perovskite solar cells, Nat. Mater. 13, 897 (2014).

[33] D. Shi, V. Adinolfi, R. Comin, M. Yuan, E. Alarousu, A. Buin, Y. Chen, S. Hoogland, A. Rothenberger, K. Katsiev, Y. Losovyj, X. Zhang, P. A. Dowben, O. F. Mohammed, E. H. Sargent, and O. M. Bakr, Low trap-state density and long carrier diffusion in organolead trihalide perovskite single crystals, Science $\mathbf{3 4 7}$, 519 (2015).

[34] E. Hecht, Optics, 4th ed. (Addison-Wesley, San Francisco, 2002).

[35] Y.-F. Tsay, B. Bendow, and S. S. Mitra, Theory of the temperature derivative of the refractive index in transparent crystals, Phys. Rev. B 8, 2688 (1973).
[36] X. Liu and J. K. Furdyna, Optical dispersion of ternary II-VI semiconductor alloys, J. Appl. Phys. 95, 7754 (2004).

[37] M. Fox, Optical Properties of Solids, 2nd ed. (Oxford University Press, New York, 2010).

[38] A. Poglitsch and D. Weber, Dynamic disorder in methylammoniumtrihalogenoplumbates (II) observed by millimeter-wave spectroscopy, J. Chem. Phys. 87, 6373 (1987).

[39] P. J. L. Hervé and L. K. J. Vandamme, Empirical temperature dependence of the refractive index of semiconductors, J. Appl. Phys. 77, 5476 (1995).

[40] Y. Rakita, S. R. Cohen, N. K. Kedem, G. Hodes, and D. Cahen, Mechanical properties of $\mathrm{APbX}_{3}\left(\mathrm{~A}=\mathrm{Cs}\right.$ or $\mathrm{CH}_{3} \mathrm{NH}_{3}$; $\mathrm{X}=\mathrm{I}$ or $\mathrm{Br}$ ) perovskite single crystals, MRS Commun. 5, 623 (2015).

[41] C. Katan, A. D. Mohite, and J. Even, Entropy in halide perovskites, Nat. Mater. 17, 377 (2018).

[42] M. Keshavarz, M. Ottesen, S. Wiedmann, M. Wharmby, R. Küchler, H. Yuan, E. Debroye, J. A. Steele, J. Martens, N. E. Hussey, M. Bremholm, M. B. J. Roeffaers, and J. Hofkens, Tracking structural phase transitions in lead-halide perovskites by means of thermal expansion, Adv. Mater. 31, 1900521 (2019).

[43] T. Soma, J. Satoh, and H. Matsuo, Thermal expansion coefficient of GaAs and InP, Solid State Commun. 42, 889 (1982).

[44] Y. Okada and Y. Tokumaru, Precise determination of lattice parameter and thermal expansion coefficient of silicon between 300 and 1500 K, J. Appl. Phys. 56, 314 (1984).

[45] T. Yamada, T. Aharen, and Y. Kanemitsu, Near-Band-Edge Optical Responses of $\mathrm{CH}_{3} \mathrm{NH}_{3} \mathrm{PbCl}_{3}$ Single Crystals: Photon Recycling of Excitonic Luminescence, Phys. Rev. Lett. 120, 057404 (2018).

[46] N. Onoda-Yamamuro, T. Matsuo, and H. Suga, Calorimetric and IR spectroscopic studies of phase transitions in methylammonium trihalogenoplumbates (II), J. Phys. Chem. Solids 51, 1383 (1990).

[47] A. I. Ekimov, F. Hache, M. C. Schanne-Klein, D. Ricard, C. Flytzanis I. A. Kudryavtsev, T. V. Yazeva, A. V. Rodina, and A. L. Efros, Absorption and intensity-dependent photoluminescence measurements on CdSe quantum dots: Assignment of the first electronic transitions, J. Opt. Soc. Am. B 10, 100 (1993).

[48] J. Tilchin, D. N. Dirin, G. I. Maikov, A. Sashchiuk, M. V. Kovalenko, and E. Lifshitz, Hydrogen-like Wannier-Mott excitons in single crystal of methylammonium lead bromide perovskite, ACS Nano 10, 6363 (2016).

[49] Y. Liu, Z. Yang, D. Cui, X. Ren, J. Sun, X. Liu, J. Zhang, Q. Wei, H. Fan, F. Yu, X. Zhang, C. Zhao, and S. F. Liu, Two-inch-sized perovskite $\mathrm{CH}_{3} \mathrm{NH}_{3} \mathrm{PbX}_{3}(\mathrm{X}=\mathrm{Cl}, \mathrm{Br}, \mathrm{I})$ crystals: Growth and characterization, Adv. Mater. 27, 5176 (2015).

[50] S. H. Wemple and M. DiDomenico, Behavior of the electronic dielectric constant in covalent and ionic materials, Phys. Rev. B 3, 1338 (1971).

[51] G. Ghosh, Temperature dispersion of refractive indices in semiconductors, J. Appl. Phys. 79, 9388 (1996).

[52] A. M. A. Leguy, P. Azarhoosh, M. I. Alonso, M. CampoyQuiles, O. J. Weber, J. Yao, D. Bryant, M. T. Weller, J. Nelson, A. Walsh, M. van Schilfgaarde, and P. R. F. Barnes, Experimen- 
tal and theoretical optical properties of methylammonium lead halide perovskites, Nanoscale 8, 6317 (2016).

[53] M. Kato, T. Fujiseki, T. Miyadera, T. Sugita, S. Fujimoto, M. Tamakoshi, M. Chikamatsu, and H. Fujiwara, Universal rules for visible-light absorption in hybrid perovskite materials, J. Appl. Phys. 121, 115501 (2017).
[54] M. Bass, E. W. Van Stryland, D. R. Williams, and W. L. Wolfe, Handbook of Optics Volume II (McGraw-Hill, New York, 1995).

[55] J. N. Zemel, J. D. Jensen, and R. B. Schoolar, Electrical and optical properties of epitaxial films of $\mathrm{PbS}, \mathrm{PbSe}, \mathrm{PbTe}$, and SnTe, Phys. Rev. 140, A330 (1965). 